\title{
A brief review of neuroimaging using functional magnetic resonance imaging (fMRI)
}

\author{
Stefan Rodic (Meds 2018), Pei Jun Zhao (Meds 2017) \\ Faculty Reviewer: Dr Ravi Menon, PhD (Department of Medical Biophysics)
}

\section{INTRODUCTION}

Since the turn of $21^{\text {st }}$ century, improvements in medical imaging have been considered one the greatest triumphs in the history of modern medicine. Advancements in magnetic resonance imaging (MRI) have allowed physicians to peer into human anatomy with ever greater resolution. MRI works through the magnetization of hydrogen protons in the body followed by short bursts of radio waves which are reemitted and subsequently detected for noninvasive neuroimaging. However, this technique was limited to forming static structural images without providing real-time information on brain activity or function.

Blood-oxygen-level-dependent (BOLD) MRI, also known as functional MRI (fMRI), overcomes these limitations and enables physicians to visualize the activity of the brain. ${ }^{1}$ Changes in blood flow act as a marker for metabolic activity and thus neuronal firing, as blood flow increases to more active regions. BOLD was first developed in 1989 and demonstrated that deoxyhemoglobin (a natural paramagnetic substance) could act as contrast agent. ${ }^{2}$ In this article, we will review the advantages and limitations of fMRI, while introducing some developments in the field.

\section{INDICATIONS}

Currently, fMRI is mainly used for research purposes but there are a growing number of specialized clinical indications. For example, prior to neurosurgery fMRI may be used to map critical areas of the brain such as the language centres. ${ }^{3}$ It can also be used to assess brain function of patients with seizures. ${ }^{4}$ Some studies indicate that fMRI remains promising for use as a detection tool for the diagnosis of early Alzheimer's disease. ${ }^{5,6}$ This technology may also have wide-ranging applications for common psychiatric disorders. Patients with major depressive disorder, schizophrenia, attention-deficit hyperactivity disorder and autism were distinguished from the resting state of controls using BOLD imaging. ${ }^{7-10}$

However, brain activity is often variable, so clinical interpretation given the patient's context is essential. Clinicians should also consider the effect of sedation, medications and anxiety on the hemodynamic response and subsequent signal. fMRI can also be dangerous for people with pacemakers and metal valves. Nevertheless, as a fairly young technology, fMRI and its variants have the potential to become a gold standard imaging tool in neuro-medicine. ${ }^{1}$

\section{ADVANTAGES}

When compared to other non-invasive functional imaging techniques, the central advantage of fMRI is its high spatial resolution in the millimeter and potentially submillimeter range. ${ }^{11,12}$
Its ability to distinguish between smaller regions of activity is superior to positrom emission tomography (PET) and much greater than magnetoencephalography (MEG) or electroencephalography (EEG), which have spatial resolution on the order of centimeters. ${ }^{13}$ Although seemingly insignificant, such a difference in resolution is vast considering that a few square millimeters of grey matter may contain millions of neurons, constituting billions of synaptic connections.

fMRI can also detect activity deep inside the brain in three dimensional space, while EEGs generally reflect global neural activity on the cortical surface. Compared to PET, BOLD imaging does not require radioactive contrast agents or metabolites and provides higher temporal resolution. ${ }^{14}$ Thus, it is a relatively safe imaging tool, even for children and pregnant women. Along with advancements in computational software, multivariate analysis in fMRI allows many small units of the brain to be tracked in unison, without simply averaging the activity of whole brain regions together. ${ }^{1}$ Instead of having large areas of average brain activity, the use of these three-dimensional pixels (voxels) allows for greater spatial resolution and reduced noise.

The critical assumption that hemodynamic response indicates neuronal activity has been questioned, yet there is a great deal of evidence that supports the validity of blood-oxygen-dependent imaging. Electrode implants have confirmed that neuronal activation correlates with BOLD MRI recordings. ${ }^{15}$ Similarly, recent advances in optogenetics have allowed the activation of specific neuronal circuits that have also been found to match the fMRI signal. ${ }^{16,17} \mathrm{Al}$ though the scanners themselves are costly, they are common and the same platform can be used for both functional and anatomic imaging. ${ }^{14}$

\section{LIMITATIONS}

Despite being correlated with the electrical activity of neurons, fMRI is an indirect imaging method. Often studies are able to demonstrate correlation of a brain region with a given task or condition but cannot establish causation. Subtle small-scale changes in neuronal activity likely go undetected as they do not induce a significant change in blood flow, while routine hemodynamic variations may generate noise. ${ }^{14}$ fMRI cannot distinguish the number of neurons firing. A hypothetical circuit may have excitatory and inhibitory signals that cancel out, but this would only be detected as generalized neuronal activity.

Hemodynamic responses are inherently slower than electrochemical ones, which can be detected by MEG/EEG in the millisecond range. ${ }^{13}$ The temporal resolution of fMRI being on the order of 
$2-3$ seconds means that the technique is not truly real-time. ${ }^{18}$ Moreover, there is generally a trade-off between spatial and temporal resolution since it takes more time to acquire images with smaller voxels. ${ }^{14}$

In addition, the interpretation of fMRI is statistically demanding. The use of different statistical techniques can lead to subjective interpretations. Correction for multiple comparisons is absolutely necessary to reduce false positives. One humorous study demonstrated false-positive brain activity in a dead salmon when multiple comparison corrections were not made. ${ }^{19}$

Finally, there are currently few studies for practical clinical use, although research is actively being conducted in this area. To obtain higher resolution, MRIs with stronger magnetic fields are being developed. Although the genotoxic effects of a static magnetic field have been demonstrated at the cellular level, they are thought to be negligible. ${ }^{20}$

\section{NEW DEVELOPMENTS}

The basis of fMRI is to track brain function using a surrogate marker that can be detected by MRI. Thus, one avenue being explored has been to develop newer and safer intravenous contrast agents that allow sharper resolution and faster image acquisition compared to the oxygenation state of hemoglobin used by BOLD MRI. However, the benefit of these agents has not been firmly established in humans yet.

From a clinical perspective, clinician-scientists are also employing fMRI in new ways to help patients. For example, researchers at the Robarts Institute (Schulich School of Medicine \& Dentistry, Western University) are using fMRI to communicate with patients in vegetative states. ${ }^{21}$ Moreover, fMRI has the potential to become a diagnostic test to assess patients with hypoxic-ischemic brain injury, perhaps even providing a new method to determine brain death.

Another development is to use multiple modalities simultaneously to map activity in the brain. One of these combinatorial approaches is the EEG-fMRI.22 Researchers have been able to use fMRI to guide EEG signal acquisition to map the electrical activity of neurons in three-dimensional space. The challenge with multimodal imaging techniques arises when trying to find a suitable method to synthesize and interpret information from the two very different imaging tools.

\section{CONCLUSION}

Since it was first described by Ogawa et al, fMRI technology has rapidly developed as a noninvasive method to map brain activity. While it remains for the most part a research tool, fMRI has great potential to become widely adopted in clinical medicine. Already, practical applications such as localizing key brain regions prior to surgery exist. With advancements in MRI technology and increases in computational power, fMRI will be an important player in the field of neuroimaging in the future.

\section{REFERENCES}

1. Smith K. Brain imaging: fMRI 2.0. Nature. 2012;484:24-6.

2. Ogawa S, Lee TM, Kay AR, Tank DW. Brain magnetic resonance imaging with contrast dependent on blood oxygenation. P Natl Acad Sci USA. 1990;87:9868-72.

3. Zhang D, Johnston JM, Fox MD, et al. Preoperative sensorimotor mapping in brain tumor patients using spontaneous fluctuations in neuronal activity imaged with functional magnetic resonance imaging: initial experience. Neurosurgery. 2009;65:226-36.

4. Up to Date [Internet]. Wolters Kluwer, 2014. Clinical presentation and diagnosis of brain tumours. Available from: http://www.uptodate.com/ contents/clinical-presentation-and-diagnosis-of-brain-tumors

5. Dai Z, Yan C, Wang Z, et al. Discriminative analysis of early Alzheimer's disease using multi-modal imaging and multi-level characterization with multi-classifier (M3). Neuroimage. 2012;59:2187-95.

6. Supekar K, Menon V, Rubin D, Musen M, Greicius MD. Network analysis of intrinsic functional brain connectivity in Alzheimer's disease. PLoS Comput Biol. 2008;4:e1000100.

7. Craddock RC, Holtzheimer PE, 3rd, Hu XP, Mayberg HS. Disease state prediction from resting state functional connectivity. Magn Reson Med. 2009;62:1619-28.

8. Shen H, Wang L, Liu Y, Hu D. Discriminative analysis of resting-state functional connectivity patterns of schizophrenia using low dimensional embedding of fMRI. Neuroimage. 2010;49:3110-21.

9. Anderson JS, Nielsen JA, Froehlich AL, et al. Functional connectivity magnetic resonance imaging classification of autism. Brain. 2011;134:3742-54.

10. Zhu CZ, Zang YF, Cao QJ, et al. Fisher discriminative analysis of resting-state brain function for attention-deficit/hyperactivity disorder. Neuroimage. 2008;40:110-20.

11. Chen W, Ugurbil K. High spatial resolution functional magnetic resonance imaging at very-high-magnetic field. Top Magn Reson Imaging. 1999;10:63-78.

12. Wu PH, Tsai PH, Wu ML, et al. High spatial resolution brain functional MRI using submillimeter balanced steady-state free precession acquisition. Med Phys. 2013;40:122304.

13. Meyer-Lindenberg A. From maps to mechanisms through neuroimaging of schizophrenia. Nature. 2010;468:194-202.

14. Crosson B, Ford A, McGregor KM, et al. Functional imaging and related techniques: an introduction for rehabilitation researchers. J Rehabil Res Dev. 2010;47:7-34.

15. Kim DS, Ronen I, Olman C, Kim SG, Ugurbil K, Toth LJ. Spatial relationship between neuronal activity and BOLD functional MRI. Neuroimage. 2004;21:876-85.

16. Kahn I, Desai M, Knoblich U, et al. Characterization of the functional MRI response temporal linearity via optical control of neocortical pyramidal neurons. J Neurosci. 2011;31:15086-91.

17. Lee JH, Durand R, Gradinaru V, et al. Global and local fMRI signals driven by neurons defined optogenetically by type and wiring. Nature. 2010;465:788-92.

18. Posse S, Ackley E, Mutihac R, et al. Enhancement of temporal resolution and BOLD sensitivity in real-time fMRI using multi-slab echovolumar imaging. Neuroimage. 2012;61:115-30.

19. Bennett CM, Wolford GL, Miller MB. The principled control of false positives in neuroimaging. Soc Cogn Affect Neurosci. 2009;4:417-22. 
20. Hartwig V, Giovannetti G, Vanello N, Lombardi M, Landini L, Simi S. Biological Effects and Safety in Magnetic Resonance Imaging: A Review. Int J Environ Res Public Health. 2009;6:1778-98.

21. Naci L, Owen AM. Making every word count for nonresponsive patients. JAMA Neurol. 2013;70:1235-41.
22. Huster RJ, Debener S, Eichele T, Herrmann CS. Methods for simultaneous EEG-fMRI: an introductory review. J Neurosci. 2012;32:605360 .

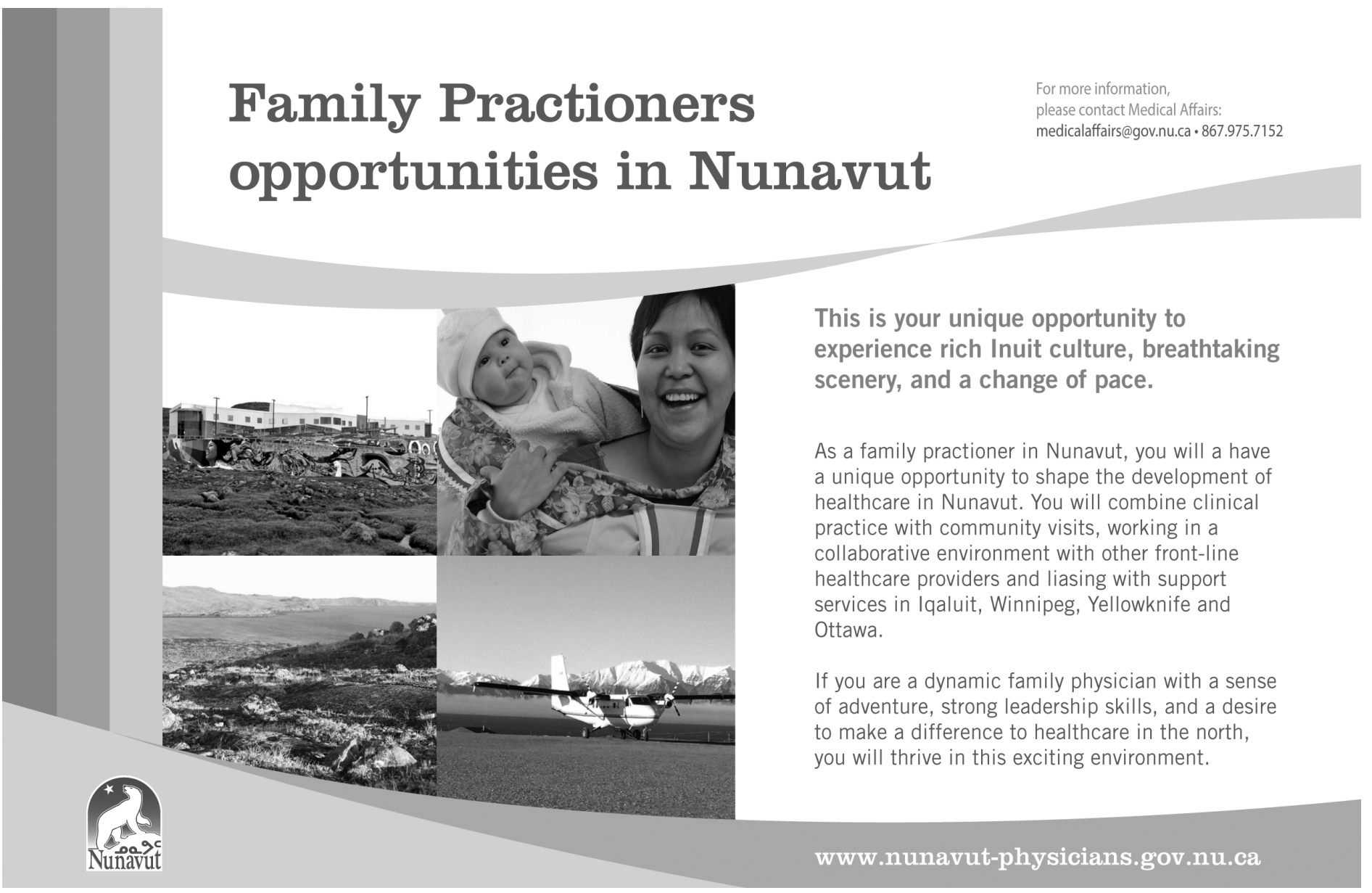

